\title{
hangares para ALITALIA
}

RICCARDO MORANDI, ingeniero

Sinopsiss Los hangares de Alitalia en el aeropuerto de Fiumicino, de Roma, se han proyectado teniendo en cuenta las técnicas y normas estéticas más modernas, las condiciones especiales de uso, la coordinación ejecutiva y de personal, el empleo adecuado de materiales y el funcionalismo
y economia óptimos.

E1 resultado de este estudio tan exhaustivo ha sido un total éxito en todos los aspectos.

La parte esencial de esta obra es la cubierta. Está constituida por una serie de nervios, cada uno de los cuales consta de cuatro elementos, uno de unión y tres pretensados, cuya solidarización da por resultado un conjunto curvo con concavidades hacia arriba y sustentado por tirantes

halla comprimido aún en el caso de que la cubierta se someta a la sobrecarga maxima prevista. La cubierta tiene una serie de huecos, cerrados con vidrieras, que facilitan la iluminación trales se sostienen suspendidas de la cubierta.

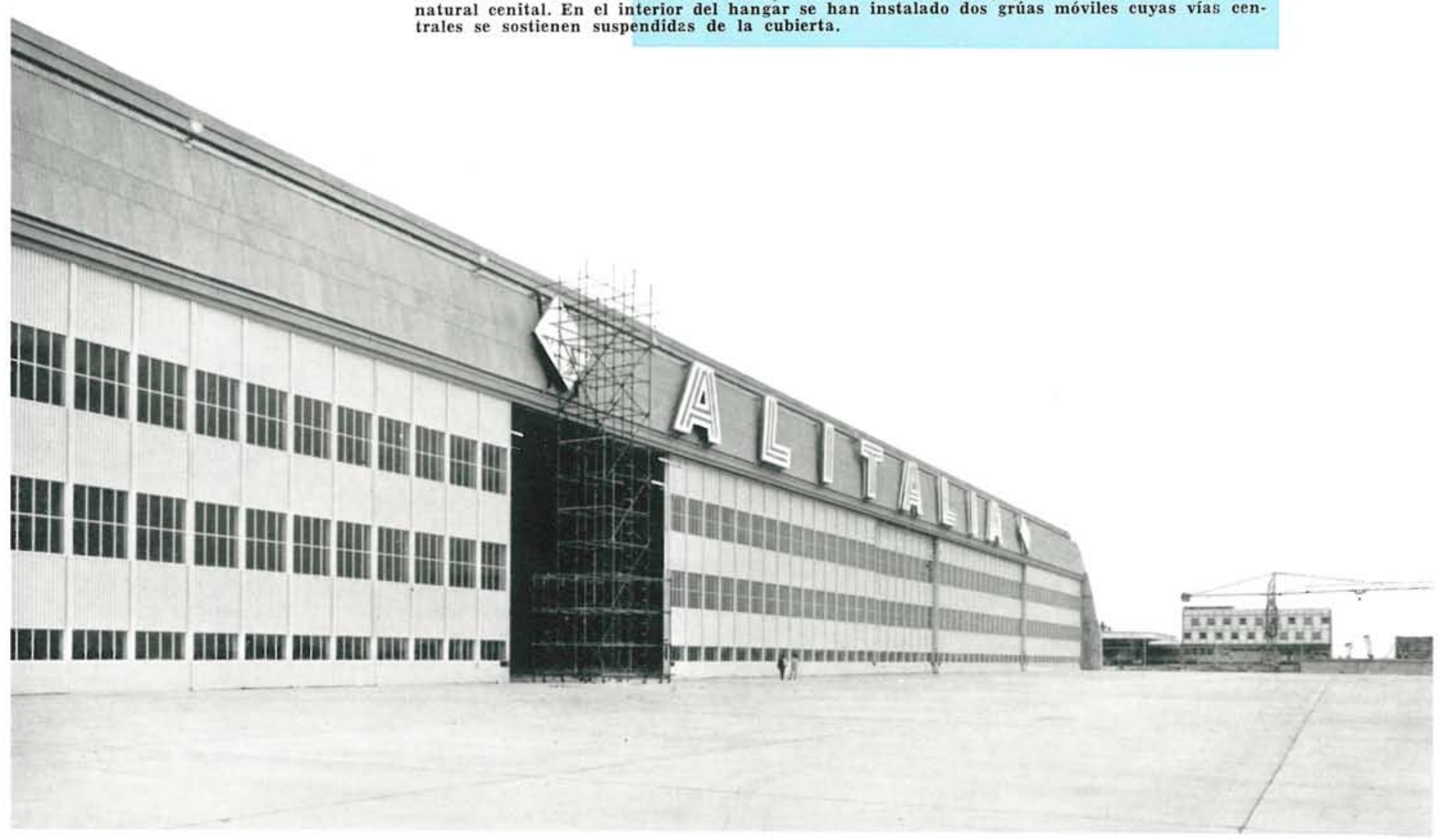




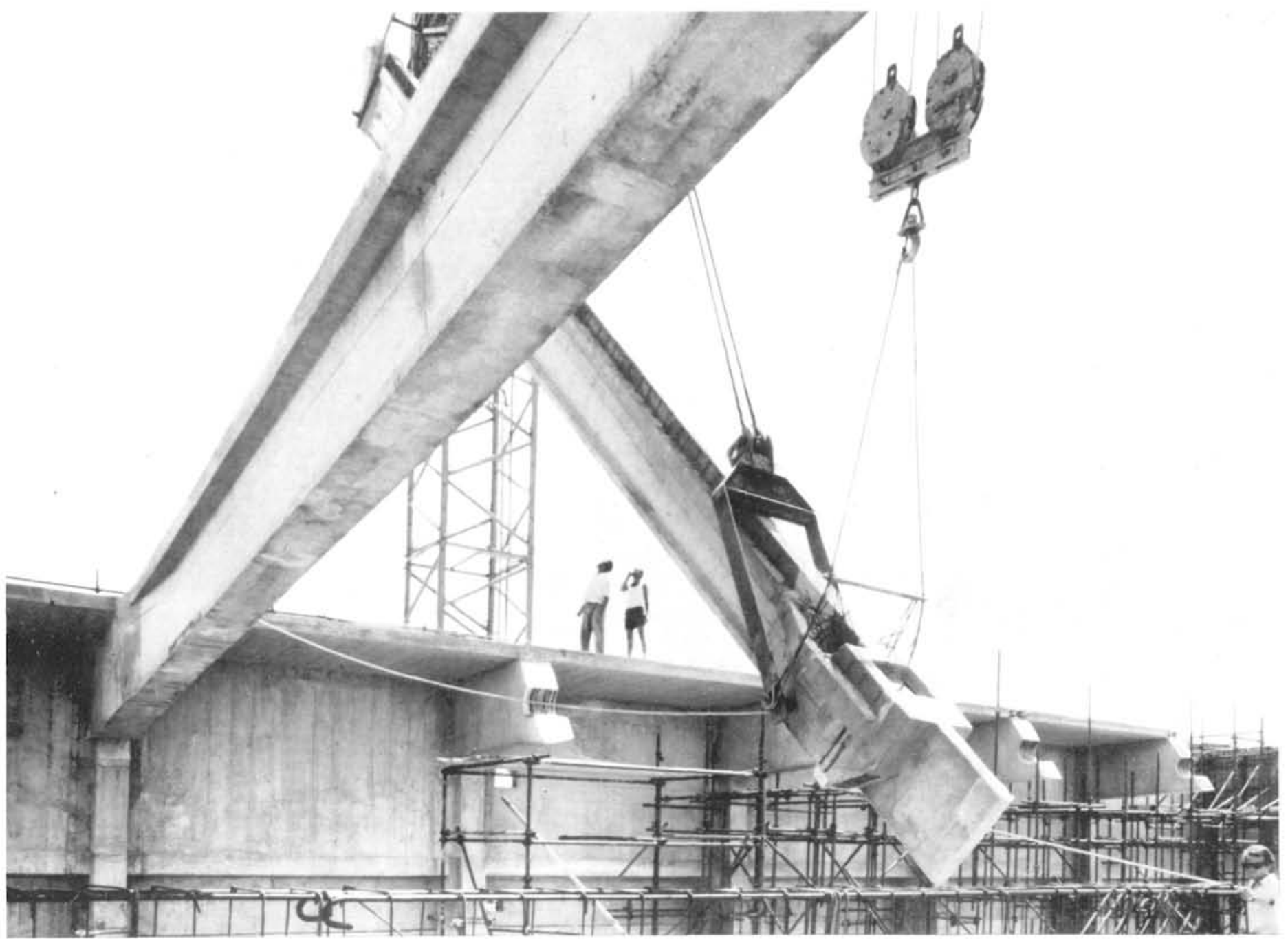

Colocación de un nervio.

Taller de prefabricación.

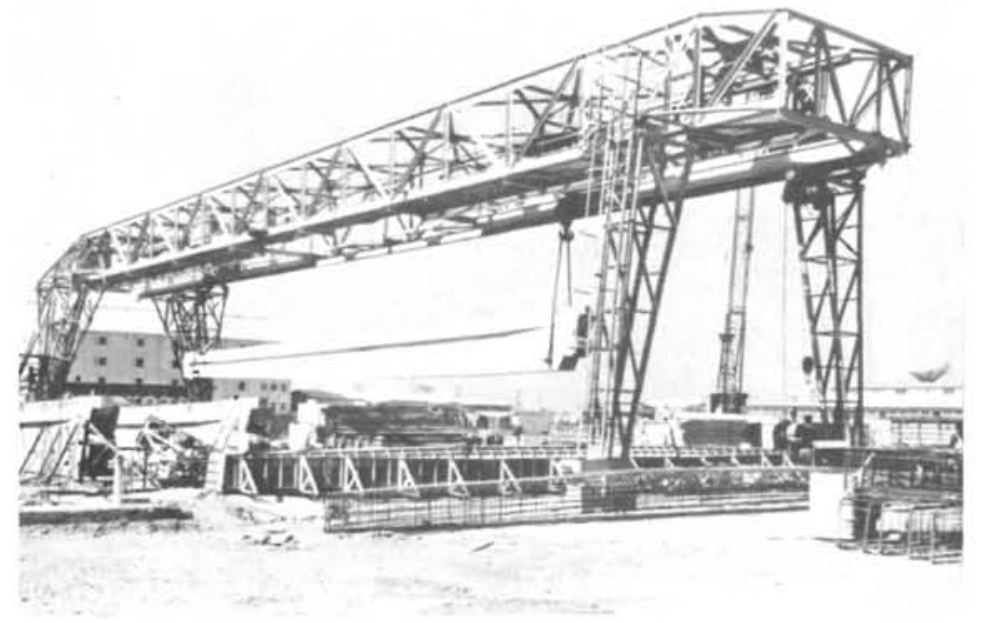

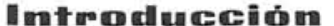

Un Centro para conservación y reparación de aviones de la compañía Alitalia, en el aeropuerto de Roma, Fiumicino (Italia), cuyo concurso para el proyecto de ejecución se hizo público en 1960, se halla actualmente en vías de terminación.

Como resultado de dicho concurso, en el que la empresa propietaria daba gran libertad a los concursantes para redactar los proyectos que mejor podían adaptarse a las condiciones mínimas básicas exigidas, el autor de este trabajo fue encargado de redactar el proyecto definitivo del conjunto de edificios que debían construirse. La solución adoptada, moderna, de hormigón armado y pretensado, presentó sus dificultades de coordinación, tanto en el orden técnico como en el económico y de ejecución.

Dado el complejo y la multitud de detalles de ejecución que debían proyectarse, fue imprescindible la creación de un grupo de colaboradores que se dedicase intensa y exclusivamente a este proyecto. 


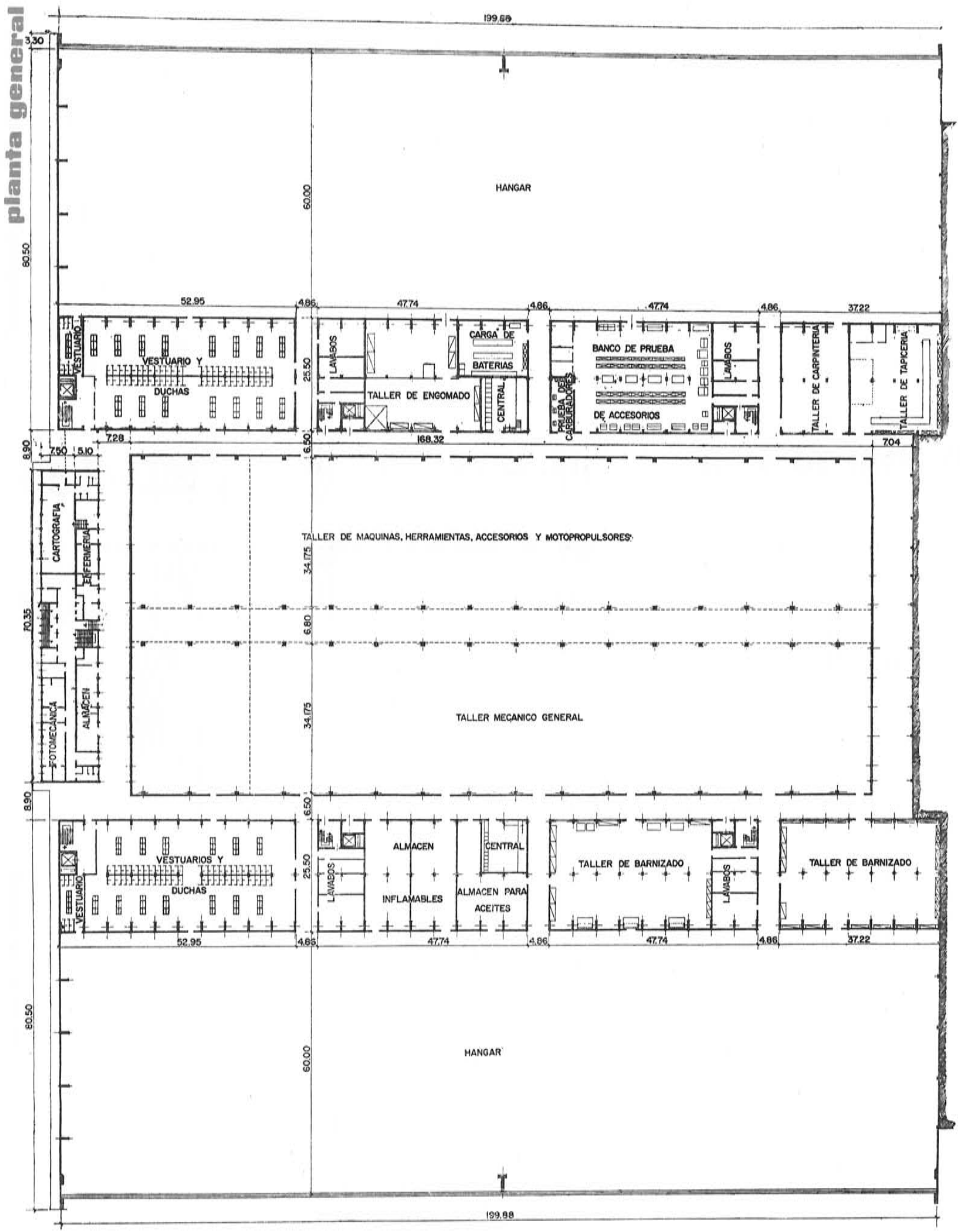



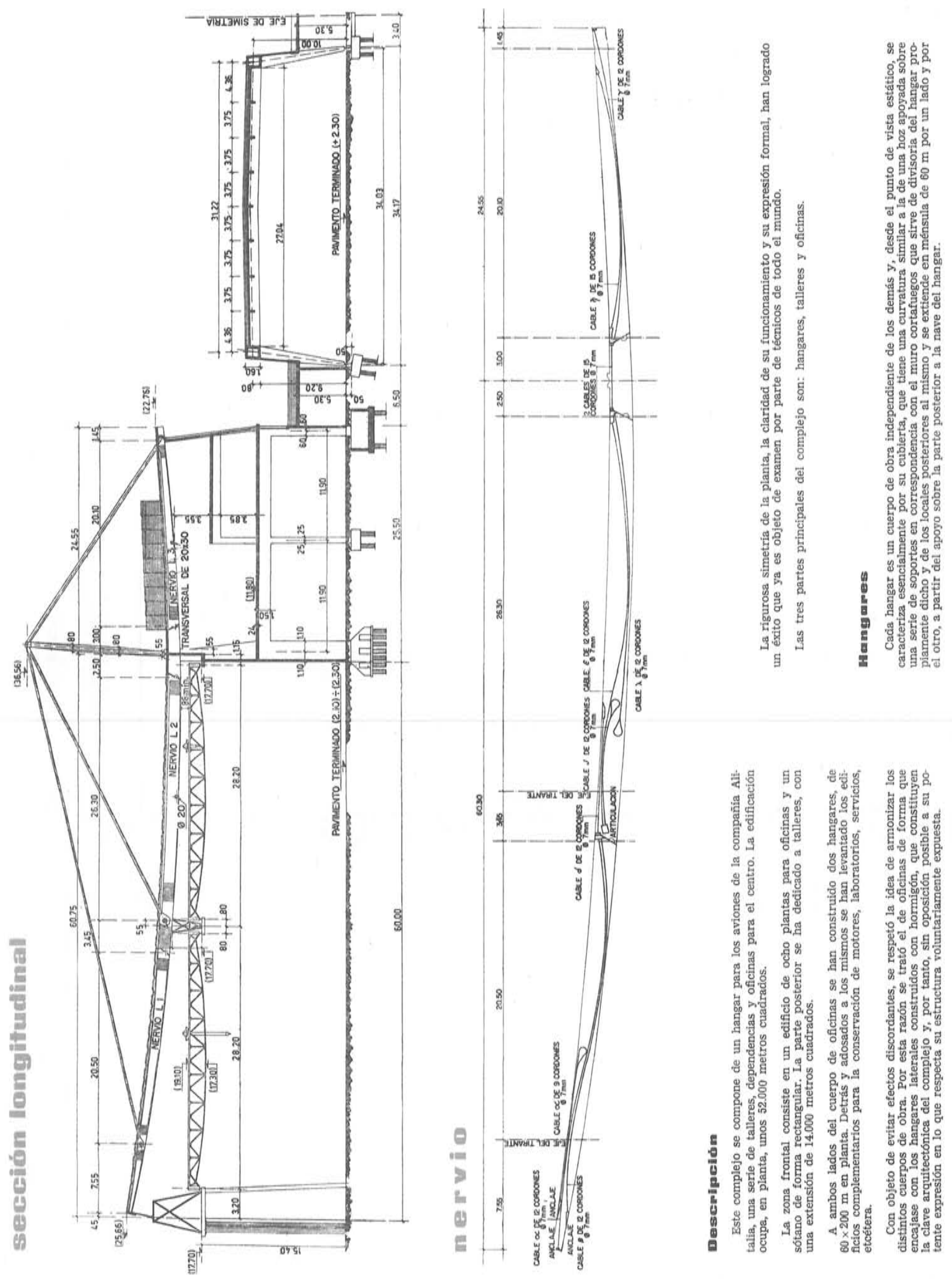
Los montantes de los locales posteriores se hallan so metidos a potentes esfuerzos transmitidos por los nervios, en voladizo, de la cubierta; otro tanto le sucede a los soportes que se encuentran en correspondencia con el muro cortafuegos. E1 muro posterior de los locales se halla sometido a esfuerzos tangenciales provocados por el corrimiento diferencial posible de la cubierta.

Todos los soportes se han hormigonado en obra $y$ apoyado sobre pilotes tipo Fran $\mathrm{ki}$, de $0,50 \mathrm{~m}$ de diámetro, de unos $15 \mathrm{~m}$ de longitud e hincados en un banco de arena. La capacidad máxima de cada pilote es de 70 toneladas.

Los cimientos de los soportes del edificio posterior al hangar se componen de cajones de hormigón armado que se han rellenado de are na, ya que se hallan sometidos a esfuerzos de tracción como consecuencia de los efectos del voladizo de la cubierta.

La cubierta está constituida por una serie de nervios paralelos, de hormigón pre tensado, unidos entre sí por medio de elementos planos, de hormigón, nervados, cada uno de los cuales tiene un hueco rectangular, que se ha cerrado con láminas transpa rentes de plástico con objeto de mejorar la iluminación natural.

\section{Cu biำ dell thering}

Cada nervio o elemento principal, resistente, de la cubierta, se ha subdividido en tres partes por medio de juntas articuladas, que el autor

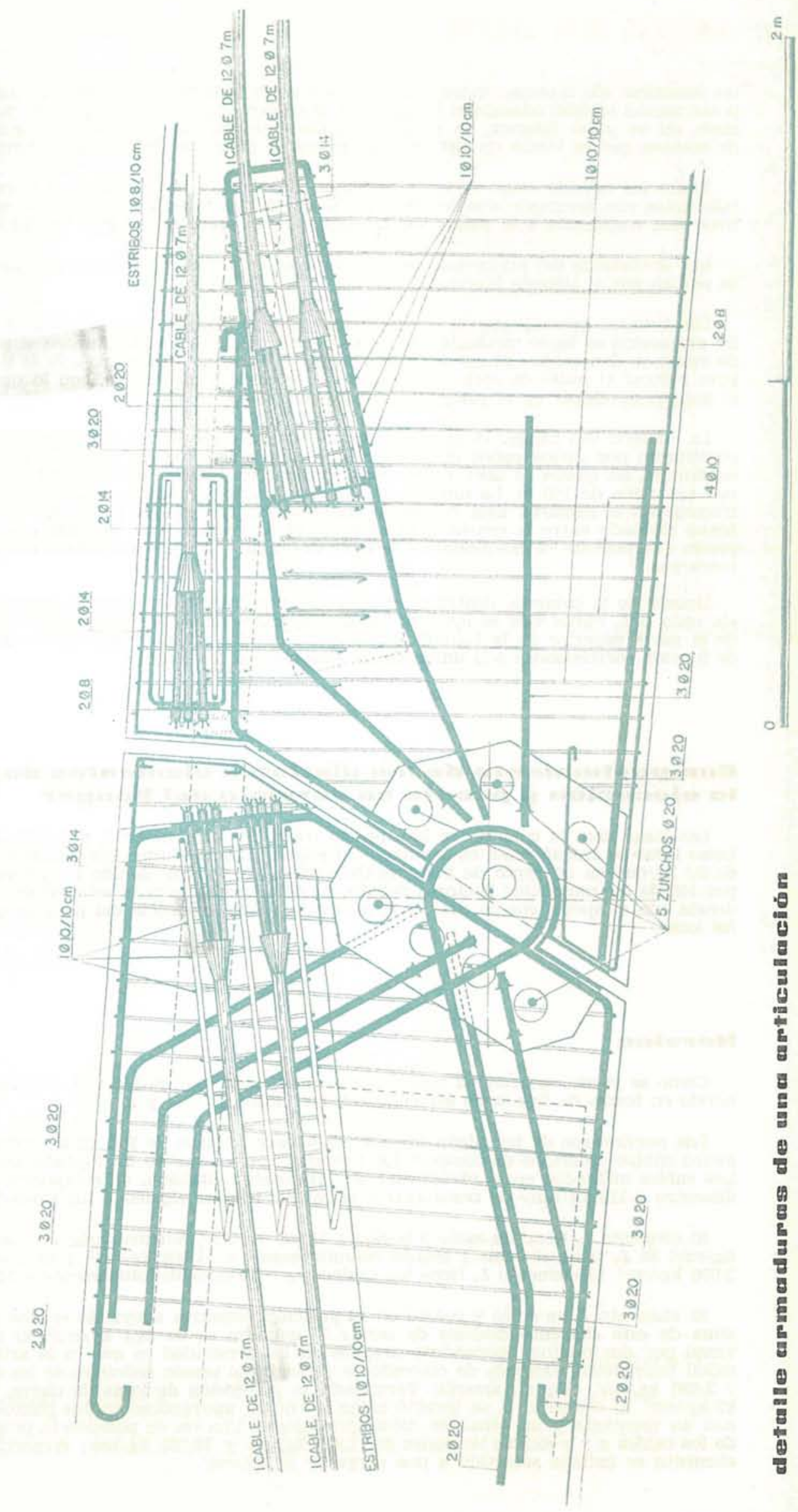


las denomina «de cápsula». Estos nervios se sostienen, aparte del soporte del muro cortafuegos y el muro posterior del edificio adosado al hangar, por dos tirantes que, pasando por encima de una torre o antena se unen, en su parte inferior, en puntos singulares de los tres elementos de que se compone cada nervio, de manera que se forme un sistema estáticamente determinado de simple composición.

Tanto los nervios como la torre de suspensión y las losas que cierran la cubierta, son elementos prefabricados con hormigón dosificado a razón de $350 \mathrm{~kg}$ de cemento por metro cúbico. El cemento utilizado tiene una resistencia a la rotura de $730 \mathrm{~kg} / \mathrm{cm}^{2}$ y el acero es del tipo Aq. 50 (italiano).

Las armaduras del pretensado están formadas con alambre de $7 \mathrm{~mm}$ de diámetro. Su puesta en tensión se realizó por el sistema Morandi.

Los tirantes que soportan la cubierta son cables metálicos de acero de gran resistencia $\left(165 \mathrm{~kg} / \mathrm{mm}^{2}\right)$. $\mathrm{Su}$ protección se logró mediante una vaina de hormigón, colocada durante el período de tesado al objeto de evitar su fisuración-ya que esto daría lugar al deterioro del acero con el tiempo-, y, principalmente, para reducir el radio de acción de las tensiones en el propio acero, con lo que se conseguiría disminuir el peligro de entrar en el campo de rotura por fatiga.

La cubierta del hangar es completamente independiente de la estructura del gran portalón de entrada, constituido por varios paños que se corren eléctricamente sobre ruedas y poleas apoyadas en las guías superiores, las cuales se unen a una viga continua en celosía, de acero dulce y de sección trapezoidal, en una extensión de $100 \mathrm{~m}$. La función de esta viga es la de absorber los esfuerzos del aire que el portalón transmite a la cubierta. Esta celosía se ha revestido con placas onduladas de eternite, mientras que el hueco formado entre la celosía y la cubierta se ha cerrado con hormigón y un dispositivo de biela y pistón que permite el movimiento relativo entre viga y cubierta debido a cambios de temperatura y sobrecargas.

Debajo de la cubierta, dentro del hangar, se han instalado dos grúas pórtico, de 5 toneladas de potencia cada una, cuyas vías se apoyan: las dos laterales sobre el muro trasero cortafuegos y en la celosía de la parte superior de la fachada, y las dos centrales, en vías suspendidas de la cubierta cuyos puntos de fijación corresponden a la unión de los tirantes con los nervios.

\section{Eomportaniento de los distimtos alememtes durante Ia ejecucion g puesta em servicio del hangar}

Las losas para la cubierta se han prefabricado. Su peso es de $1 \mathrm{t}$, y sus dimensiones, $4,98 \times 1,55 \mathrm{~m}$. Estas losas se transforman en continuas al soldar las armaduras, admitiéndose, para su cálculo, una carga de $30 \mathrm{~kg} / \mathrm{cm}^{2}$ en concepto de impermeabilización, $120 \mathrm{~kg} / \mathrm{cm}^{2}$ debido a sobrecargas accidentales y un 20 por 100 de los momentos flectores debidos al peso propio para la solicitación de la flexión en correspon. dencia con el apoyo, con lo cual se tiene en cuenta la influencia del peso propio y de la continuidad de las losas.

\section{Marvios}

Como se puede apreciar en una de las figuras, cada uno de los tres elementos de que se compone el nervio en forma de hoz se ha señalado con las iniciales $L_{1}, L_{2}$ y $L_{3}$

Los nervios son de hormigón armado, dosificado a razón de $350 \mathrm{~kg}$ de cemento (tipo $730 \mathrm{~kg} / \mathrm{cm}^{2}$ ) por metro cúbico de áridos de composición ternaria, con lo que se ha logrado un hormigón de $450 \mathrm{~kg} / \mathrm{cm}^{2}$ Los cables utilizados en el pretensado, de fabricación italiana, se componen de alambres de $7 \mathrm{~mm}$ de diámetro y $170 \mathrm{~kg} / \mathrm{mm}^{2}$ de resistencia a la rotura, tesados siguiendo los procedimientos Morandi.

El elemento $L_{1}$ tiene un cable $\beta$ tesado a $11.500 \mathrm{~kg} / \mathrm{cm}^{2}$, mientras que el $\alpha$ se tesó parcialmente a 7.600 $\mathrm{kg} / \mathrm{cm}^{2}$. El $L_{2}$ tiene el cable $\lambda$ tesado definitivamente a $11.100 \mathrm{~kg} / \mathrm{cm}^{2}$, y el $\varepsilon$ con un esfuerzo parcial de $3.000 \mathrm{~kg} / \mathrm{cm}^{2}$. El elemento $L_{3}$ tiene los cables $\eta$ y $\gamma$ tesados definitivamente a $11.400 \mathrm{~kg} / \mathrm{cm}^{2}$.

El elemento $L_{3}$ se elevó y colocó en su posición definitiva apoyando en dos puntos. La solicitación máxima de este elemento después de cerrar la cubierta es de $83,4 \mathrm{~kg} / \mathrm{cm}^{2}$. El elemento $L_{2}$ también se levantó por dos puntos, apoyándose después en la extremidad en que va la articulación y en un castillete móvil provisional. Después de colocado se procedió al tesado definitivo de los cables $\delta, \varepsilon, J$ a $10.700,5.800$ y $2.600 \mathrm{~kg} / \mathrm{cm}^{2}$, respectivamente. Terminada la colocación de losas de cierre, la carga resultante fue de $62 \mathrm{~kg} / \mathrm{cm}^{2}$. El elemento $L_{1}$ se levantó como los otros, apoyándose en dos puntos y se mantuvo en posición con un montante y un caballete móvil provisional. Una vez en posición se procedió a completar el tesado de los cables $\alpha$ y $\gamma$ con las tensiones de $11.600 \mathrm{~kg} / \mathrm{cm}^{2}$ y $10.700 \mathrm{~kg} / \mathrm{cm}^{2}$, respectivamente. El hormigón del elemento se hallaba sometido a una carga de $81 \mathrm{~kg} / \mathrm{cm}^{2}$. 


\section{cunbiemate del edifficio de talleres}

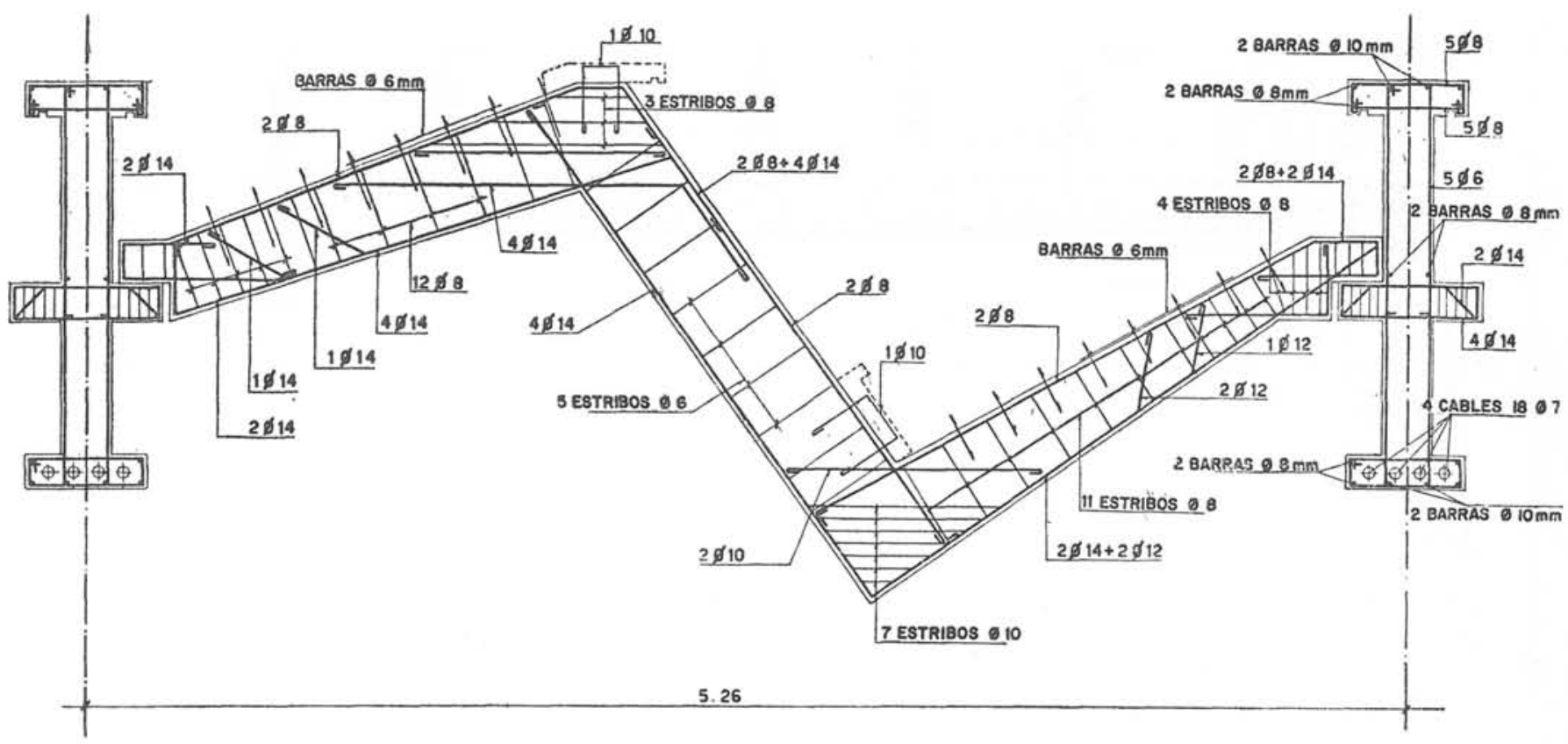

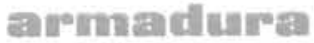

Colocados ya los nervios se procedió al montaje de los tirantes que, pasando por la parte superior de las torres, anclan, cada extremidad, en los puntos previstos en cada nervio. El tesado de los tirantes se dio por terminado cuando los nervios se despegaron de los soportes provisionales que los mantenía en posición. La tensión final de los mismos fue del orden de $5.000 \mathrm{~kg} / \mathrm{cm}^{2}$. Finalmente, se procedió a dar la tensión definitiva a los cables de pretensado de los nervios, alcanzando los $11.500 \mathrm{~kg} / \mathrm{cm}^{2}$. El hormigón de los nervios, en su fase final, resultó ser de $173 \mathrm{~kg} / \mathrm{cm}^{2}$ como máximo.

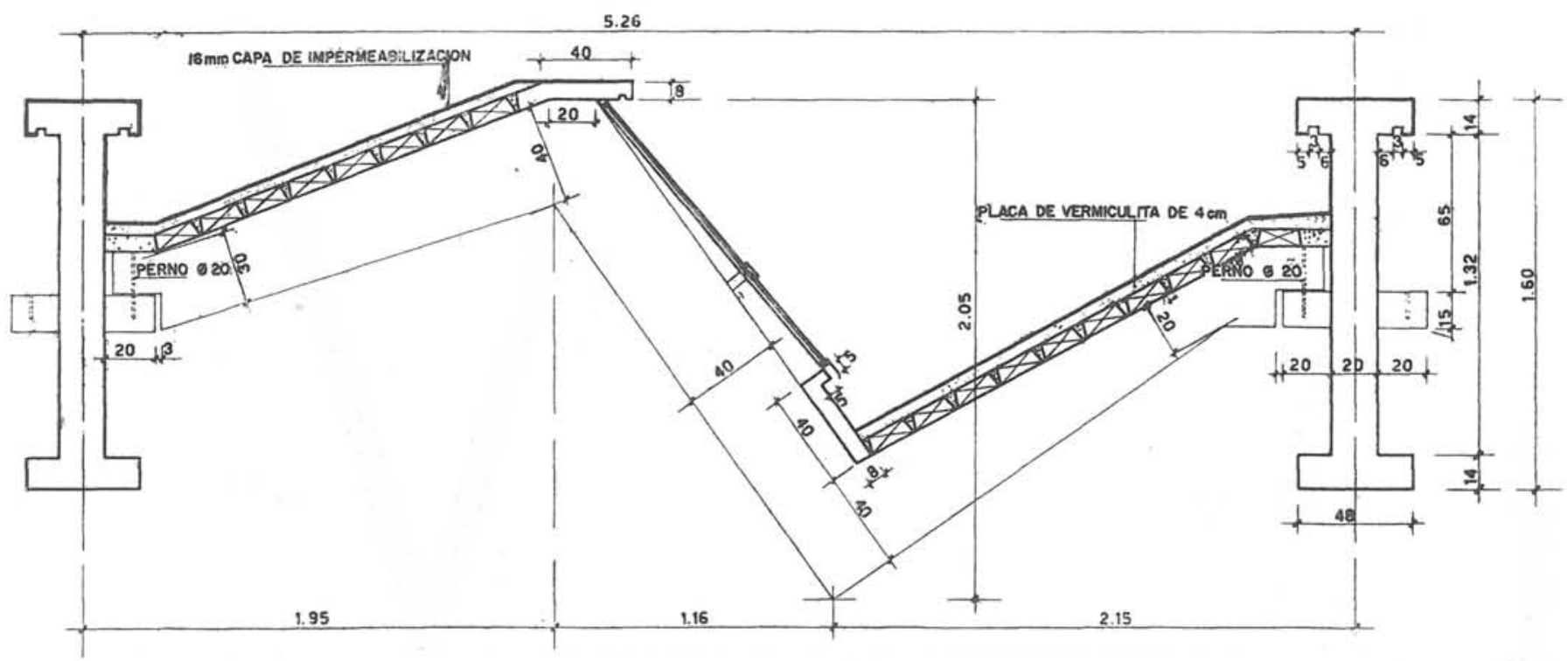




\section{pórnticeo de tallleres}
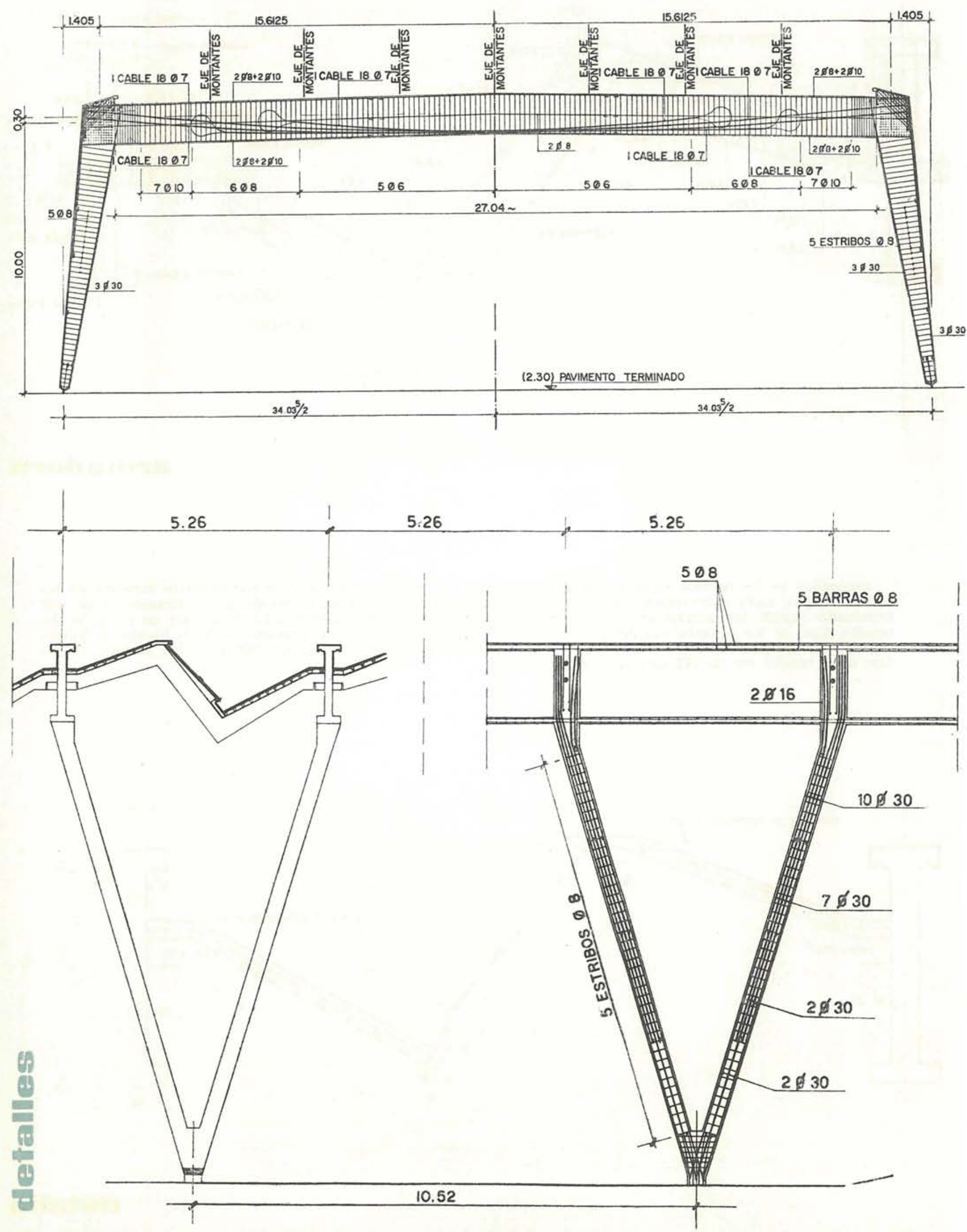


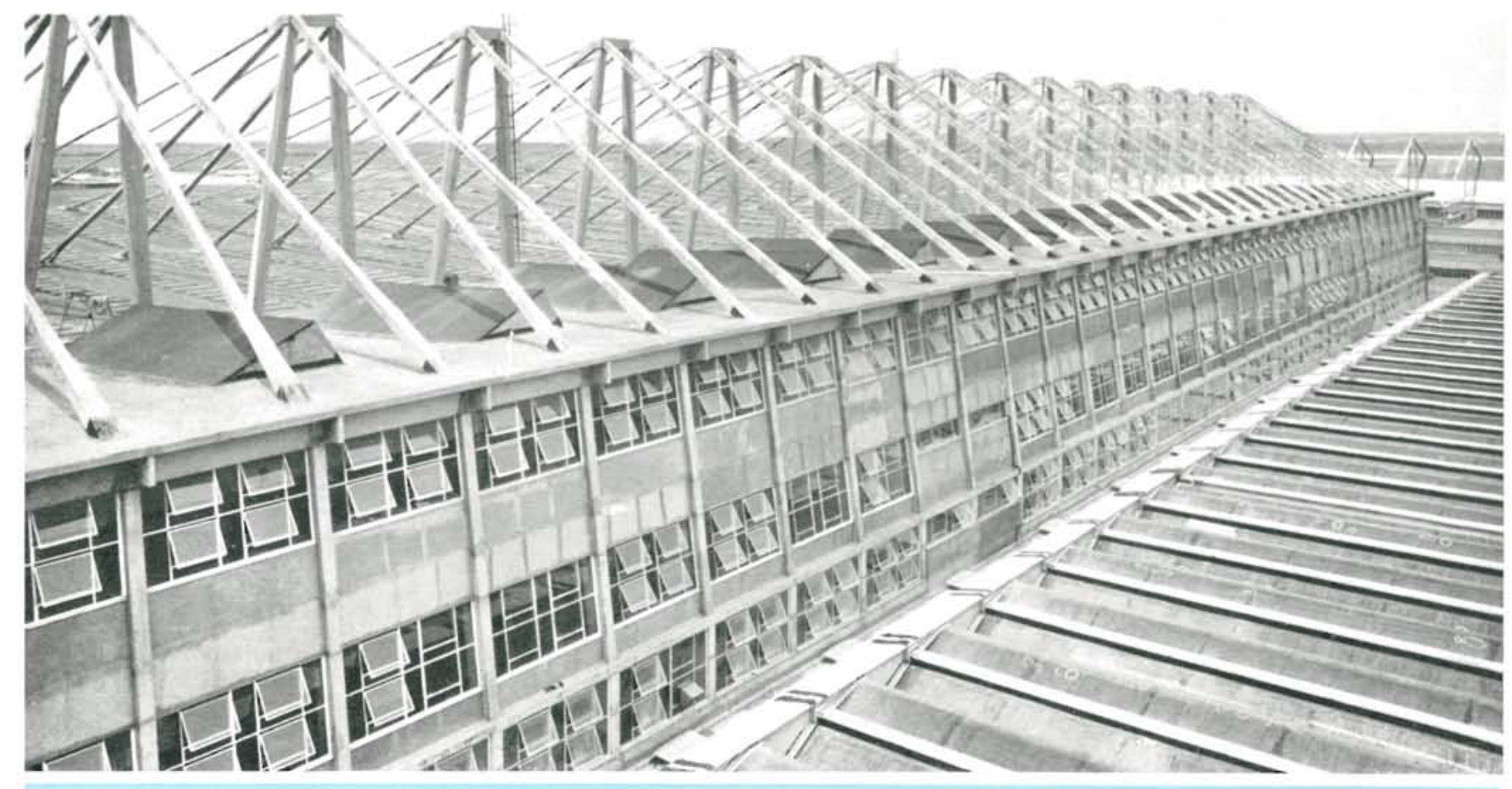

Zona posterior de un hangar.

Vista interior de un hangar.

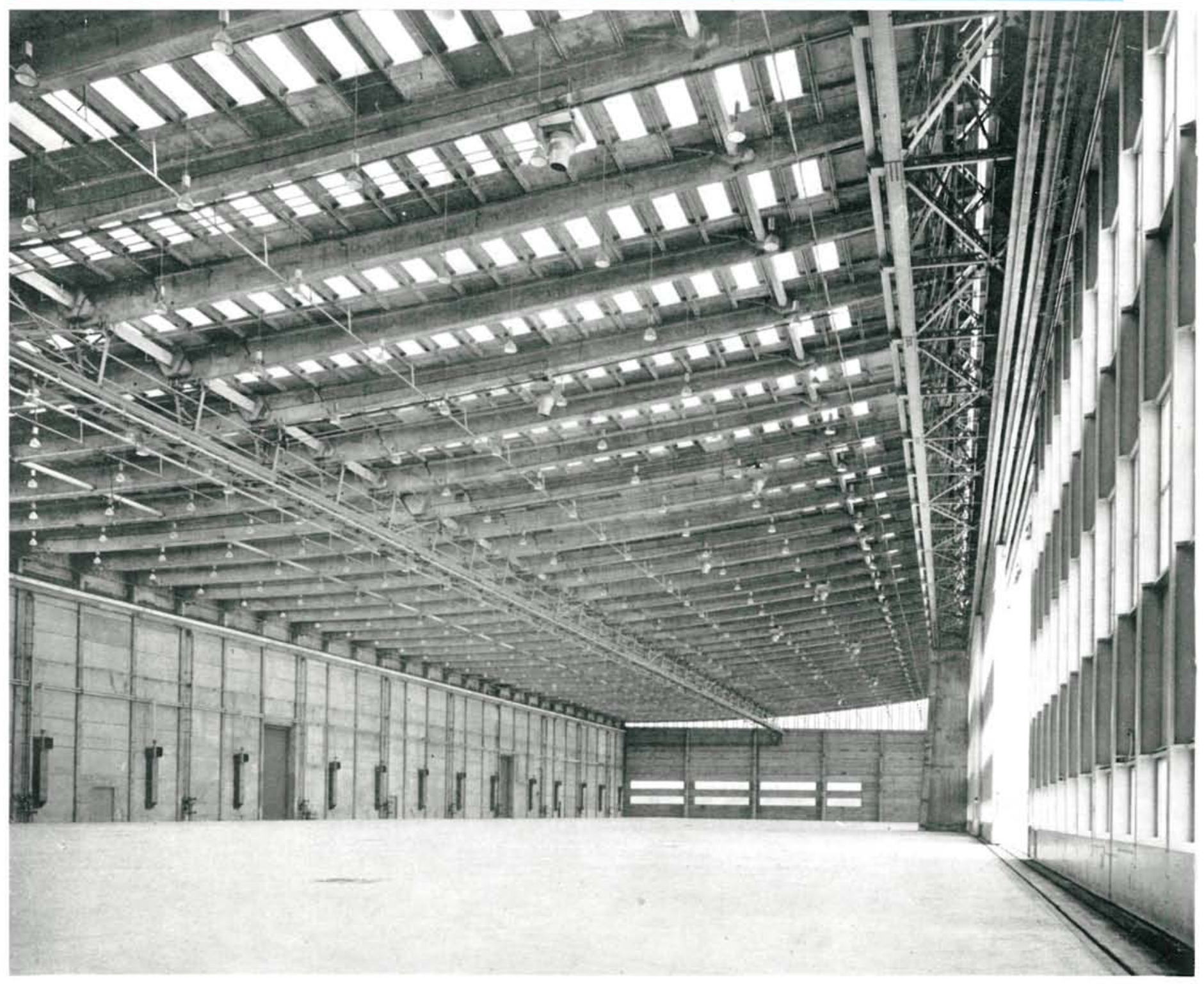



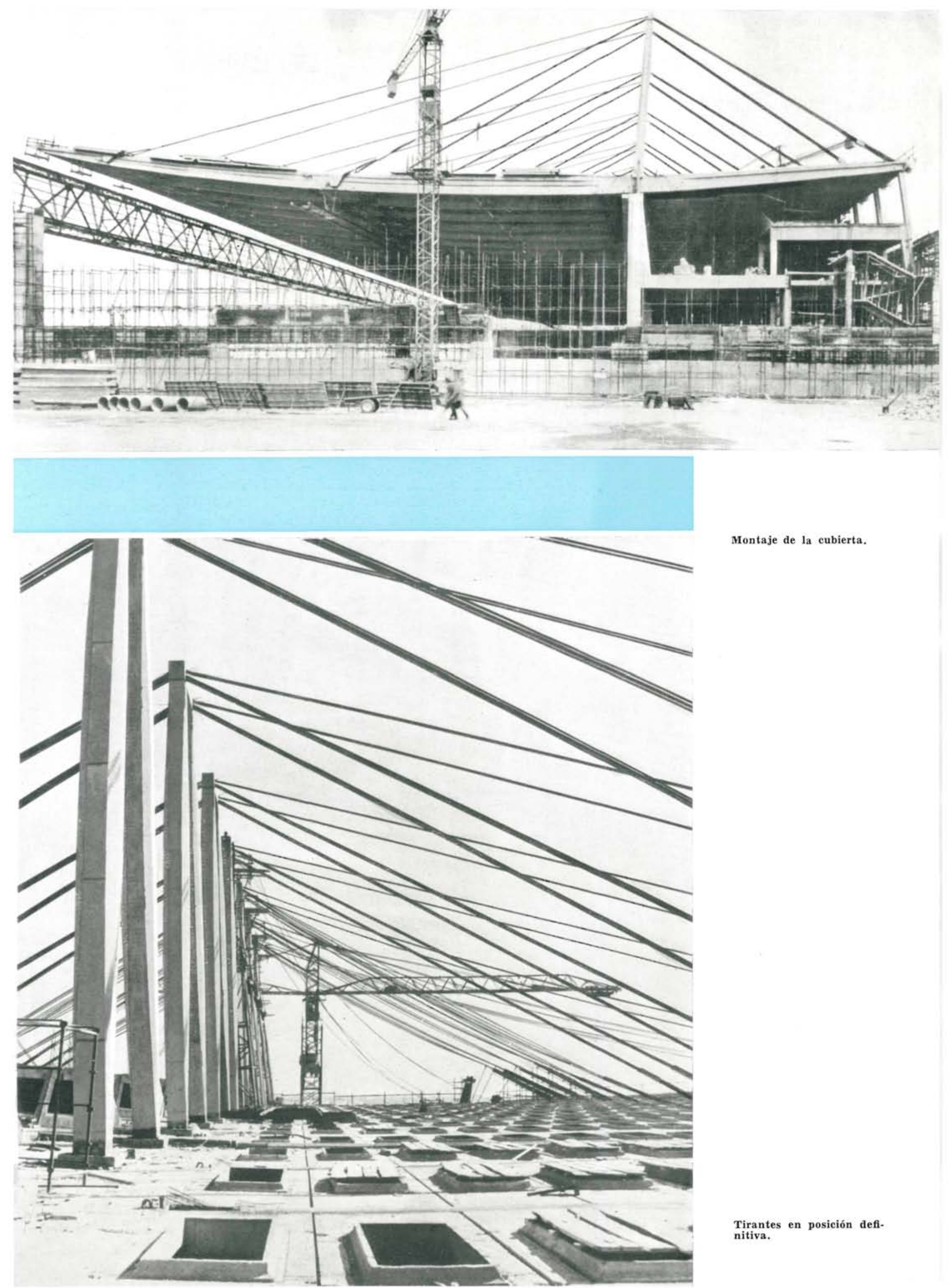

Montaje de la cubierta.

Tirantes en posición definitiva. 


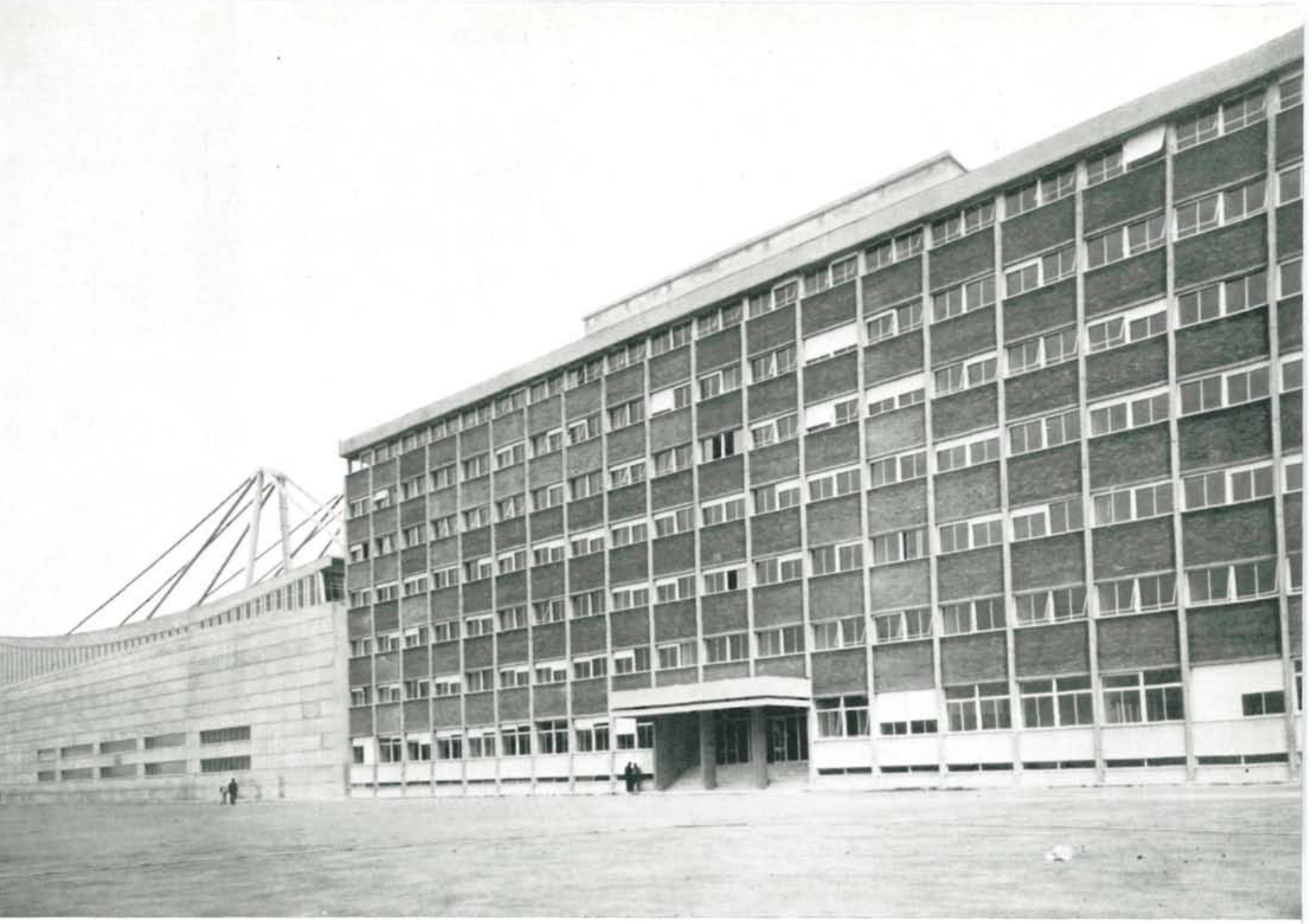

Fachada principal de oficinas.

Los tirantes se revistieron con una vaina de hormigón, pero evitando se produjeran efectos de flexión debidos al propio peso y retesando los tirantes, de tal forma que al final se hallase la vaina sometida a compresiones aun en el caso de sobrecargas accidentales.

\section{Prouelom fingut}

Terminada la obra se encargó de su prueba, antes de entrar en servicio, a la Facultad de Ingeniería de la Universidad de Pisa. Entre los datos recogidos en dicha prueba, el valor resultante para el módulo de elasticidad del hormigón fuə de $350.000 \mathrm{~kg} / \mathrm{cm}^{2}$. La deformación residual, despreciable, es del orden del 5 por 100 de la máxima prevista para las sobrecargas. El período de la vibración propia del complejo ha resultado notablemente inferior a un segundo. 


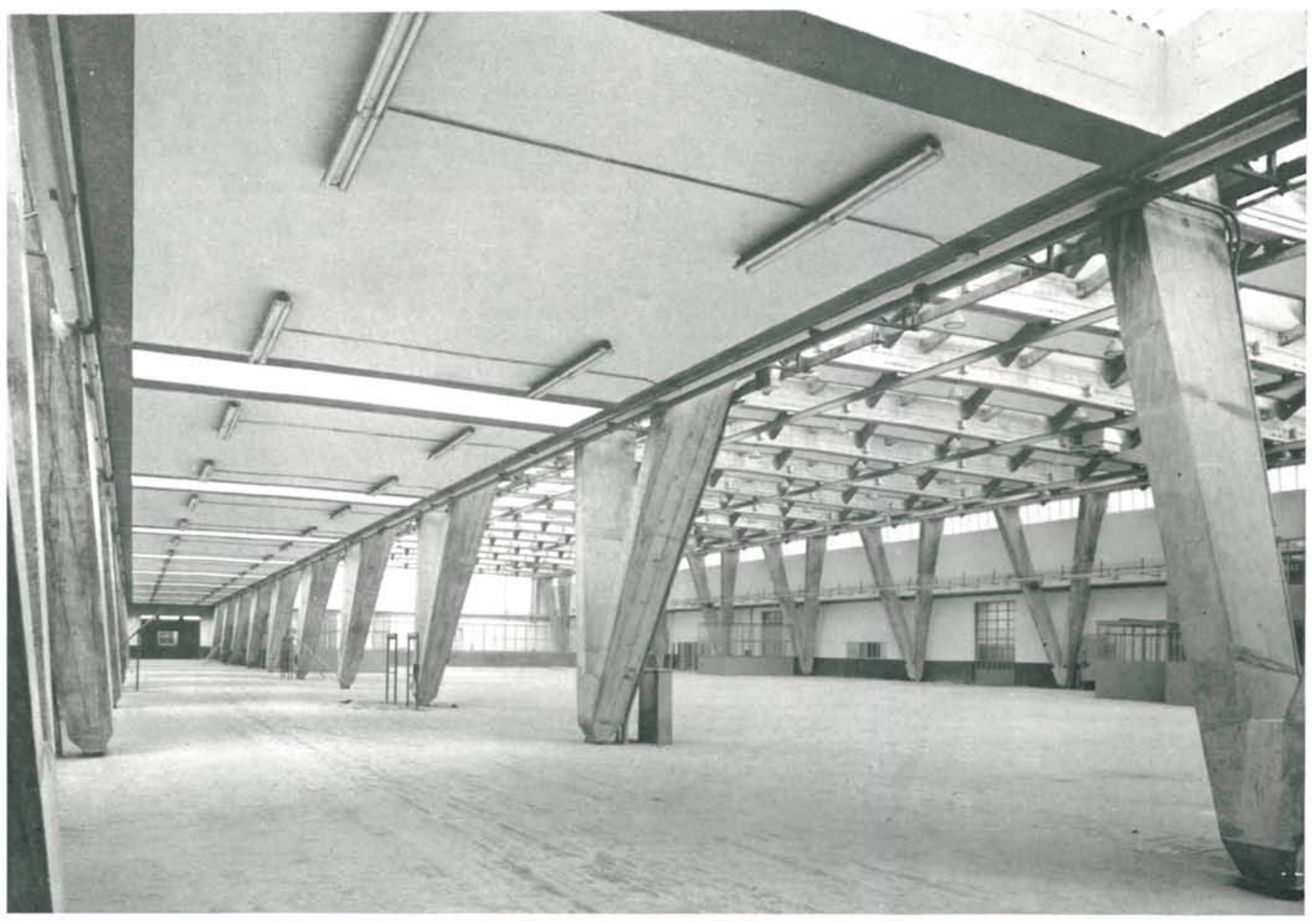

Corredor entre los dos talleres,-Soportes oblicuos en forma de V.

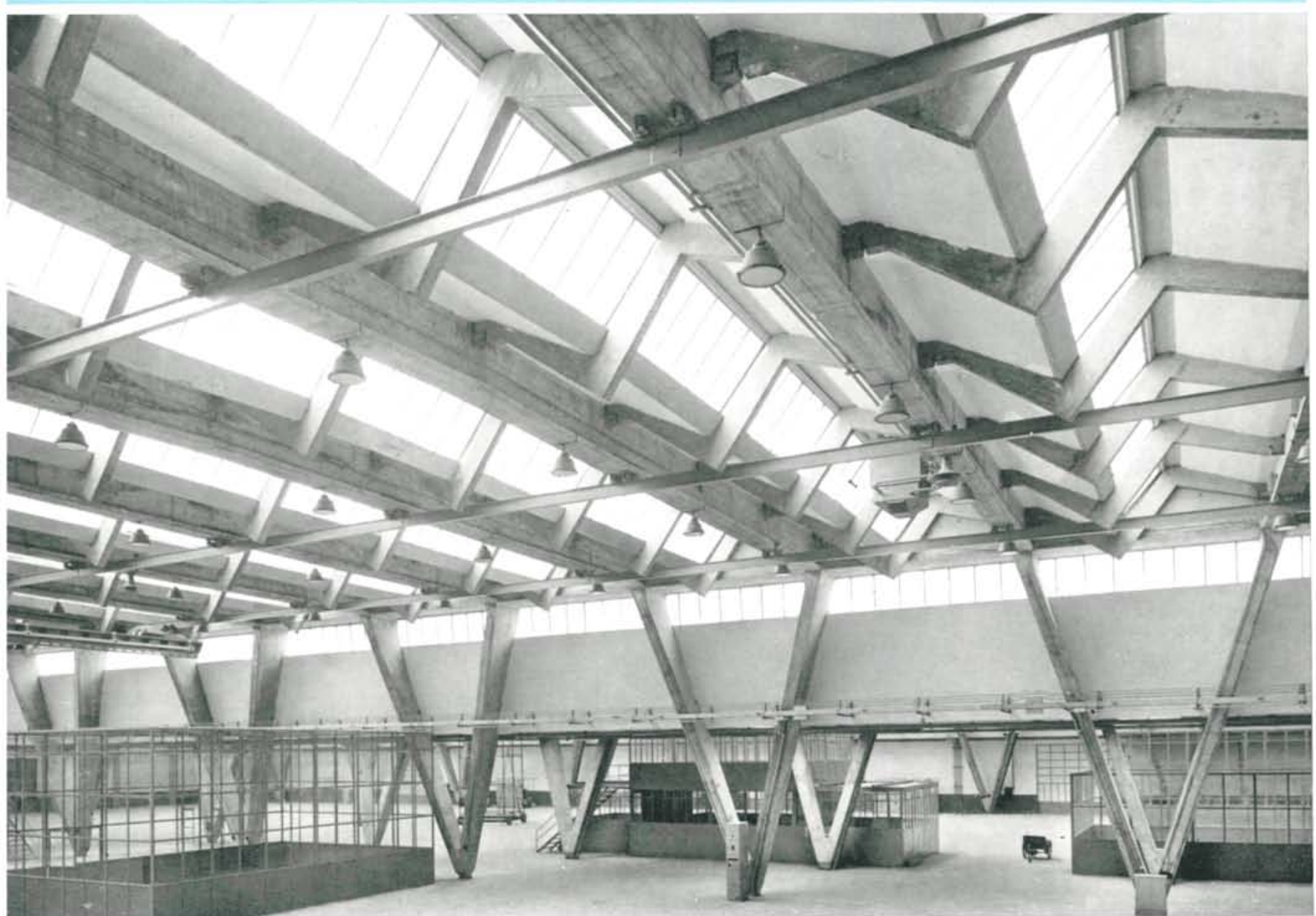




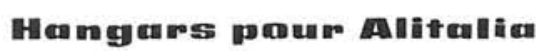

Riccardo Morandi, ingénieur.

Les hangars d'Alitalia à l'aéroport Fiumicino, de Rome, ont été conçus selon les techniques et les normes esthétiques les plus modernes, en se pliant aux conditions spéciales de l'usage auquel ils sont destinés, à la coordination exécutive et au personnel, à l'emploi adéquat des matériaux, au caractère fonctionnel et à l'économie optima.

Le résultat de cette étude exhaustive a été un succès complet sous tous les aspects.

La partie essentielle de cet ouvrage a été la couverture. Elle est formée par une série de nervures, dont chacune se compose de quatre éléments, un d'union et trois précontraints, dont la solidarisation a pour résul. tâ un ensemble courbe, légèrement concave, relevé par-devant et supporté par des tirants métalliques re. vêtus de béton, de telle façon que celui-ci soit comprimé même au cas où la couverture serait soumise à la surcharge maximale prévue.

La couverture a une série de baies, munies de verrières, qui permettent l'éclairage naturel zénithal. A l'intérieur des hangars ont été installées deux grues mobiles dont les voies centrales sont suspendues à la couverture.

\section{Anĩ}

Riccardo Morandi, engineer.

The Alitalia hangars, at the Fiumicino Airport, Rome, have been designed according to most modern technical and aesthetic ideas, and also taking account of the special functional and staff requirements, availability of materials, and optimum economic efficiency.

The result of a most exhaustive initial research has been the total success of the final project.

The outstanding feature is the roof. It consists of a series of ribs, each in four parts, one to join, and the other three prestressed. Together they provide a curved structure, with concavities facing upward. They are supported by metal tie members, which have been embedded in concrete, so that the tie is under compression even if the roof is under the heaviest estimated overload.

The roof has a number of openings, closed with glass slabs, which provide natural illumination from the top. Inside the hangar there are two moveable cranes, whose central rails hang from the roof.

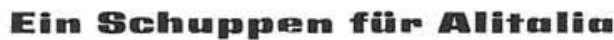

Riccardo Morandi, Ingenieur.

Der Schuppen der Alitalia auf dem Flugplatz Fiumicino in Rom wurde sowohl nach den modernsten technischen und ästhetischen Gesichtspunkten als auch unter Beachtung der funktionellen Erfordernisse entworfen.

Das Resultat solch eingehender Studien war in jeder Hinsicht ein voller Erfolg.

Den wesentlichsten Teil dieses Bauwerkes bildet das Dach. Es besteht aus einer Reihe von Nerven, die jeweils aus einem Verbindungselement und drei vorgespannten Elementen bestehen. Durch die Verbindung dieser Nerven entsteht ein nach oben gewölbtes Ganzes, das von metallischen Zugbändern gehalten wird. Letztere wurden mit Beton so verkleidet, dass dieser sich auch dann unter Druck befindet, wenn das Dach der höchstzulässigen Belastung ausgesetzt ist.

In das Dach sind eine Reihe von Glasfenstern eingelassen, die eine natürliche Beleuchtung von oben ermö. glichen. Im Innern des Schuppens sind zwei Fahrkrähne eingebaut, deren Mittelschienen an der Decke befestigt sind. 